\title{
Dapagliflozin Attenuates Diabetic Cardiomyopathy Through Erythropoietin Up-Regulation of AKT/JAK/MAPK Pathways in Streptozotocin- Induced Diabetic Rats
}

\author{
Nora El-Sayed \\ Sinai University \\ Yasser M. Mostafa \\ Suez Canal University \\ Noha M AboGresha \\ Suez Canal University \\ Amal A.M. Ahmed \\ Suez Canal University \\ Islam Z. Mahmoud \\ Suez Canal University \\ Norhan M. El-Sayed ( $D$ norhan.ms@pharm.suez.edu.eg ) \\ Suez Canal University https://orcid.org/0000-0002-0432-3152
}

\section{Research Article}

Keywords: Dapagliflozin, Diabetic Cardiomyopathy, Erythropoietin, AKT/JAK/MAPK pathways

Posted Date: May 13th, 2021

DOl: https://doi.org/10.21203/rs.3.rs-472044/v1

License: (c) This work is licensed under a Creative Commons Attribution 4.0 International License.

Read Full License 


\section{Abstract}

Purpose: This study was designed to investigate the mechanism of Dapagliflozin (Dapa) cardioprotection against diabetic cardiomyopathy (DCM). Structural and functional changes in the heart as well as decrease of Erythropoietin (EPO) levels were reported in DCM. EPO simultaneously activates three pathways: the Janus-activated kinase-signal transducer and activator of transcription (JAK2/STAT5), phosphatidylinositol-3-kinase-Akt (PI3K/Akt), and extracellular signal-related kinase (ERK/MAPK) cascades, that result in proliferation and differentiation of cardiac cells.

Methods and Results: DCM was induced by a high fat diet for 10 weeks followed by administration of streptozotocin. After confirmation of diabetes, rats were divided randomly to 5 groups: Group 1; normal control group, Group 2; untreated diabetic group and Groups (3-5); diabetic groups received Dapa daily $(0.75 \mathrm{mg}, 1.5$ or $3 \mathrm{mg} / \mathrm{Kg}, \mathrm{p} .0)$ respectively for a month. At the end of the experiment, full anaesthesia was induced in all rats using ether inhalation and ECG was recorded. Blood samples were collected then rats were sacrificed and their heart were dissected out and processed for biochemical and histopathological studies. Untreated diabetic rats showed abnormal ECG pattern, elevation of serum cardiac enzymes, decrease EPO levels, downregulation of P-Akt, P-JAK2 and pMAPK pathways, abnormal histological structure of the heart and increase immunostaining intensity of P53 and TNF $a$ in the cardiomyocytes. Dapa in a dose dependent manner attenuated the alterations in the previously mentioned parameters.

Conclusion: The cardioprotective effect of Dapa could be mediated by increasing EPO levels and activation of P-Akt, P-JAK2 and pMAPK signalling cascades which in turn decrease apoptosis.

\section{Introduction}

Diabetic patients have twice to 4 times higher risk compared with non-diabetic people for morbidity and mortality of cardiovascular (CV) diseases. Diabetes is associated with many changes in the structure and function of myocardium; a disorder known as diabetic cardiomyopathy (DCM) [1]. More than $80 \%$ diabetic patients are or will be suffered from DCM. Moreover, about $50-80 \%$ of death in diabetic patients is because of DCM. DCM is characterized by left ventricular hypertrophy (LVH), myocardial fibrosis, dysfunctional remodelling, diastolic dysfunction, later systolic dysfunction, and eventually presented with heart failure [1]. Previous studies showed a deficiency in Erythropoietin (EPO) levels in patients with type 2 diabetes compared to non-diabetic patients [2,3]. EPO is a glycoprotein hormone that is produced by the kidney in response to hypoxia. Binding of EPO with its receptor simultaneously activates three major transduction pathways including the Janus-activated kinase- signal transducer and activator of transcription (JAK2-STAT5), phosphatidylinositol-3-kinase-Akt (PI3K-Akt), and extracellular signal-related kinase (ERK) cascades. Activation of these signalling pathways results in proliferation and differentiation of cells [4]. Although most antidiabetic drugs have been used to reduce microvascular complications of diabetes, no respectable effect was observed on macrovascular complications. However, sodium glucose transporter (SGLT2) inhibitors show significant effects not only on the depression of microvascular 
complications, but also on the depression of macrovascular complications including cardiovascular complications [5]. They have remarkable cardioprotective effect and reduce hospitalization and mortality of HF in type 2 diabetes patients [5]. Nevertheless, the mechanisms of their cardioprotective effects are not yet fully investigated. Dapagliflozin (Dapa) is a member of SGLT2 inhibitors that shows some promising benefits on diabetic patients with $\mathrm{CV}$ diseases. The present study was designed to investigate the cardioprotective effect of Dapa in rats with type 2 diabetic cardiomyopathy focusing on its effect on EPO levels and the expression of P-Akt, P-JAK2 and pMAPK signalling cascades.

\section{Materials And Methods \\ 2.1. Animals}

Fifty Wistar male rats with an average weight of $150-200 \mathrm{~g}$ were used in this study. Animals were kept under controlled environmental conditions at $\left(25 \pm 4^{\circ} \mathrm{C}\right)$ on 12 -h dark / light cycles. The animals were housed in stainless-steel cages with free access to standard balanced diet and fresh water supply. This study was carried out according to the "Guidelines of the Animal Care and Use of laboratory animals "and in parallel with the guidelines adopted by Research Committee at Suez Canal University (approval no. $201812 \mathrm{MA}_{2}$ ).

\subsection{Chemicals and drugs}

Streptozotocin (STZ) was purchased from (MP Biomedicals, LLC). Dapagliflozin was provided by AstraZeneca Company (Cairo, Egypt) and it was dissolved in normal saline.

\subsection{Induction of Type 2 diabetes mellitus in rats}

Forty Wistar rats were fed a high fat carbohydrate diet (HFD), 4 kcal\% fat (lard), 35\% carbohydrate (corn starch, sucrose, and maltodextrin) and $20 \%$ protein (casein) for 10 weeks. Rats then were fasted for 12 hours overnight and injected with freshly prepared STZ dissolved in $0.1 \mathrm{M}$ citrate buffer $(\mathrm{pH} \mathrm{4.4)}$ ) at a single dose $(30 \mathrm{mg} / \mathrm{kg}$, i.p) to induce type 2 diabetes mellitus [6]. Animals were continued HFD during the post-STZ administration period. Fasting blood glucose level using a blood sample obtained from the tail vein was measured by a glucometer after a week of administration of STZ to make sure of the stability and effectiveness of the diabetes induction. Rats with fasting blood glucose levels $>250 \mathrm{mg} / \mathrm{dl}$ were considered diabetic and included in this study.

\subsection{Experimental design}

After confirmation of diabetes, rats were randomly divided into five experimental groups; Group 1: rats were fed a normal diet during all the experiment $(51.93 \mathrm{~g}$ carbohydrate, $3.03 \mathrm{~g}$ lipid, $20.50 \mathrm{~g}$ protein and $4.17 \mathrm{~g}$ crude fibre $/ 100 \mathrm{~g}$ diet, and $3.00 \mathrm{kcal} / \mathrm{g}$ energy) and received oral administration of normal saline daily and considered normal control group, Group 2: diabetic rats received saline, Groups (3-5): diabetic rats received daily doses of Dapa $(0.75,1.5$ or $3 \mathrm{mg} / \mathrm{kg}$, p.o) respectively for 4 weeks. At the end of the experiment, full anaesthesia was induced in all rats using ethyl ether inhalation. Electrocardiography 
(ECG) of anesthetized rats from all groups was recorded. Blood samples from all rats were collected from the orbital sinus under anaesthesia just before scarification for determination of cardiac markers. Rats were sacrificed by cervical dislocation under light ether anaesthesia. After scarification, heart was dissected and weighed for determination of myocardial index (MI) and Left ventricular weight index (LVW). Cardiac tissue samples were then cut into 2 halves, one half was frozen at $-80^{\circ} \mathrm{C}$ for biochemical analysis and the other half was fixed in $10 \%$ neutral buffered formalin for histopathological and immunohistochemistry examination.

\subsection{Electrocardiography}

Electrocardiography (ECG) of all live anesthetized rats was recorded for at least 2 minute using research Biopac Data Acquisition mp150 device (BIOPAC Systems Inc., Santa Barbara, CA). Full anaesthesia was induced by ethyl ether inhalation for 30-40 seconds [7]. For each rat, lead II was recorded, in which V + electrode was inserted subcutaneously (s.c) to the left leg, V- electrode was inserted s.c to the right arm, and ground electrode was inserted s.c to the right leg. The following parameters were recorded for each ECG tracing: voltage of R (mv), depth of Q wave and QT interval prolongation (ms).

\subsection{Evaluation of myocardial index and Left ventricular weight index}

Hearts of all rats from different groups were dissected, dried on filter papers, and then weighed to determine myocardial index (MI). Myocardial index was calculated by (weight of heart to weight of body weight ratio) ${ }^{*} 100$ to determine the extent of hypertrophy because of diabetic cardiomyopathy. Then atria and right ventricles were separated and left ventricular weight (LVW) was weighed. LVW index was calculated by (LVW/heart weight ratio) *100 to confirm the induction of myocardial dysfunction.

\subsection{Determination of Cardiac Markers}

Just before scarification and under light ether anaesthesia, blood samples were collected from the orbital sinus using a clean sterile capillary tube, which was inserted into the inner cantus of the eye. The blood was then left to be clotted for 20 minutes and then centrifuged at $1195 \mathrm{~g}$ for 15 minutes. Serum samples were separated, collected, and frozen at $-20^{\circ} \mathrm{C}$ for determination of cardiac markers. Serum levels of total creatine-kinase (CK), creatine-kinase-MB Isoenzyme (CK-MB), lactate dehydrogenase (LDH) and cardiac troponin I (CTnl) were determined spectrophotometrically using ELISA kits (cat\# MBS722021, MBS008782, MBS043166 and MBS727624 respectively, BioSource company) according to manufacture instructions.

\subsection{Determination of Erythropoietin levels}

Serum EPO levels were determined quantitively using ELISA kit (cat\# MBS703724, BioSource Company) according to manufacture instructions.

\subsection{Western blot analysis}


For detection of P-Akt, P-JAK2 and pMAPK, the cardiac tissues were homogenised in cold RIBA buffer supplied with additional protease inhibitor and phosphatase inhibitor buffer to maintain protein integrity and its biological activity. Cell debris was removed by centrifugation at $16.000 \mathrm{~g}$ for 10 minutes. The supernatants were transferred to a new tube and stored at $-80^{\circ} \mathrm{C}$ for determination of protein concentration using Bradford assay. 5-20 microgram protein of each sample was mixed with $2 x$ Laemmli sample buffer (4\% sodium dodecyl sulphate, $20 \%$ glycerol, $10 \%$ 2-mercaptoethanol, $0.125 \mathrm{M}$ Tris $\mathrm{HCl}, 0.004 \%$ bromophenol blue). The $\mathrm{pH}$ was adjusted at 6.8 and the mixture was boiled at $95^{\circ} \mathrm{C}$ for $5 \mathrm{~min}$ to ensure protein denaturation then centrifuged for $10 \mathrm{~min}$ at $10.000 \mathrm{~g}$. The final supernatants were supposed to $12 \%$ sodium dodecyl sulfate polyacrylamide electrophoresis. Bio-Rad Trans -Blot turbo instrument (Bio-Rad Laboratories Ltd, Watford, UK) was used in protein blotting from gel to PVDF membrane. The membrane was then blocked to prevent nonspecific binding of antibodies in Tris-buffered saline (TBS) with Tween 20 (TBS-T) buffer and $3 \%$ bovine serum albumin (BSA) containing $5 \%$ (wt/vol) non-fat dry milk for 1 hour. The membranes were incubated with primary antibodies (rabbit monoclonal anti-Phospho-Akt (Ser473) [mAb Cat\# 4060 cell signalling technology], rabbit monoclonal anti-p38 MAPK [mAb cat\#8690, cell signalling technology] and rabbit monoclonal antiPhospho-Jak2 (Tyr1007) mAb cat \#4406 cell signalling technology. Primary antibodies were diluted (1:1000 dilution in TBS-T with 5\% nonfat milk) at $4^{\circ} \mathrm{C}$ overnight. Blots were rinsed 3-5 times with TBS-T in the HRP-conjugated secondary antibody (Goat antirabbit IgG-HRP-Ig Goat mAb Novus Biological, 1:5000 dilution) solution. All incubations were done at room temperature for 1 hour. The chemiluminescence substrate was used for visualization (Clarity ${ }^{\text {TM }}$ Western ECL substrate - BIO-RAD, USA cat\#170-5060) according to the manufacturer's instructions and the chemiluminescent signals were captured using a CCD camera-based imager.

\subsection{Histopathological Examination}

Tissue samples of each group were exposed to routine histological techniques; they were subjected to ascending grade of ethyl alcohol or dehydration, clearing in xylol for 15 minutes, embedding in paraffin wax for 1 hour. Then, they were cut at 4-6 $\mu \mathrm{m}$ thickness. Microscopic slides were stained with hematoxylin and eosin (H\&E) for general structure of the heart and Masson trichrome for detection of collagen fibres. Histopathological inspection was done using a blinded investigator. Images from the heart were captured using a digital camera (Olympus Dp25, Japan) and fibrotic area was measured using Image Pro PLUS software (Media Cybernetics, USA).

\subsection{Immunohistochemical determination of TNF alpha and P53}

For immunohistochemical analysis of TNF alpha and P53 from the cardiac tissues, all sections were deparaffinized and incubated in citrate buffer at $\mathrm{PH}(6.1)$ in an oven $\left(95^{\circ} \mathrm{C}\right)$ for 15 minutes to ensure Antigen retrieval following Vectastain $\mathrm{ABC}$ kit protocol. Endogenous peroxidase activity was quenched by diluted $\mathrm{H}_{2} \mathrm{O}_{2}(0.3 \%)$. A Vectastain rabbit blocking reagent was used to prevent nonspecific binding. 
Sections were incubated overnight with the homologous primary antibodies; for TNF alpha:(Rabbit IgG polyclonal, Cat\#GTX110520, Gene Tex laboratories ) and for P53: Rabbit IgG polyclonal, Cat\#BS0033R,Biossantibodies laboratories). Biotinylated Secondary antibody (Linker) was applied at room temperature for 30 minutes. After rinsing, $A B C$ Vectastain reagent was applied for another 30 minutes followed by the preformed streptavidin. Sections were then incubated in peroxidase substrate $\left(3,3^{\prime}\right.$ diaminobenzidine) solution for another 5 minutes. Finally, hematoxylin was used for ( $1-3$ minutes) at room temperature for counterstaining. For detection of \% area of cells immunostained with TNF alpha and $\mathrm{p} 53$, images of selected parts of heart sections were captured by means of a digital camera (Olympus Dp25, Japan) and analysed using the ImageJ software developed by the National Institute of Health (Bethesda, Maryland, USA).

\subsection{Statistical Analysis}

Results were analyzed using Statistical Package for Social Sciences (SPSS) software version 26 (SPSS Inc., Chicago, Illinois, USA). For parametric data, differences between groups were carried out using one way analysis of variance (ANOVA) followed by Bonferroni post hoc for multiple comparisons. However, nonparametric data were analyzed using Pearson chi-square test. All Data were expressed as mean \pm SD and considered statistically significant at $P \leq 0.05$.

\section{Results}

\subsection{Dapagliflozin blunted the changes of Electrocardiographic parameters in diabetic rats}

Untreated diabetic rats showed abnormal ECG pattern represented by QT interval prolongation, decrease in voltage of R, ST segment elevation, and increase in depth of $Q$ wave in a significant manner compared with control group and all other groups as shown in Table 1 and Fig. 1. However, treatment with graded doses of Dapa $(0.75,1.5$ or $3 \mathrm{mg} / \mathrm{Kg}$ ) attenuates the highly modified ECG pattern and significantly decreased the abnormalities in a dose dependent manner as following: decreased QT interval prolongation, rendered voltage of $\mathrm{R}$ to normal, decreased ST segment elevation and decreased the percent of pathological Q waves. Additionally, diabetic group treated with either 1.5 or $3 \mathrm{mg}$ Dapa showed a significant increase in voltage of $\mathrm{R}$ compared with diabetic group treated with $0.75 \mathrm{mg}$ Dapa. Furthermore, diabetic group treated with $3 \mathrm{mg}$ Dapa could reach the normal control values in All ECG parameters detected in this study. 
Table 1

Effect of graded doses of Dapagliflozin on ECG parameters. Values of (QT interval, Voltage of R and S-T segment elevation) were expressed as mean \pm SD and analyzed using one-way ANOVA followed by the Bonferroni post hoc test for multiple comparisons. Values of Depth of Q wave were expressed as percentage and analyzed using Pearson chi-square test. Superscript letters indicate a significant difference at $\mathrm{P} \leq 0.05$ for all values (a) Indicates significant differences from control rats. (b) Indicates significant differences from untreated diabetic rats. (c) Indicates significant differences from diabetic rats-treated with $0.75 \mathrm{mg}$ Dapagliflozin.

\begin{tabular}{|c|c|c|c|c|}
\hline Study groups & $\begin{array}{l}\text { QT interval } \\
\text { (ms) }\end{array}$ & $\begin{array}{l}\text { voltage of } \\
R(m v)\end{array}$ & $\begin{array}{l}\text { S-T segment } \\
\text { elevation } \\
\text { (ms) }\end{array}$ & $\begin{array}{l}\text { Depth of Q } \\
\text { wave(mv) } \\
\text { (\%) deep }\end{array}$ \\
\hline Control & $0.15 \pm 0.02$ & $0.93 \pm 0.11$ & $0.01 \pm 0.01$ & 0 \\
\hline Untreated diabetic & $0.32 \pm 0.1^{a}$ & $0.05 \pm 0.01^{a}$ & $0.29 \pm 0.08^{a}$ & $100^{\mathrm{a}}$ \\
\hline $\begin{array}{l}\text { Diabetic + } 0.75 \mathrm{mg} \\
\text { Dapa }\end{array}$ & $0.15 \pm 0.02^{b}$ & $0.32 \pm 0.13^{a}$ & $0.03 \pm 0.02^{b}$ & $83.3^{a}$ \\
\hline $\begin{array}{l}\text { Diabetic + } 1.5 \mathrm{mg} \\
\text { Dapa }\end{array}$ & $0.14 \pm 0.01^{b}$ & $0.84 \pm 0.2^{b, c}$ & $0.02 \pm 0.02^{b}$ & $0^{b}$ \\
\hline Diabetic + 3 mg Dapa & $0.12 \pm 0.01^{b}$ & $0.91 \pm 0.2^{b, c}$ & $0.01 \pm 0.02^{b}$ & $0^{b}$ \\
\hline
\end{tabular}

The changes in myocardial index, left ventricular weight index and mortality percentage were shown in Table 2. Myocardial index and left ventricular weight index were both significantly higher in untreated diabetic group compared to control group. Oral administration of graded doses of Dapa prevented significantly hypertrophy and diabetic group treated with $3 \mathrm{mg}$ Dapa could almost reach control values in both myocardial and left ventricular weight indexes. In terms of the mortality rate, control group had $0 \%$ mortality while untreated diabetic group showed $37.5 \%$ mortality. Dapa administration reduced significantly the mortality rate compared to the untreated diabetic group. 
Table 2

Effect of graded doses of Dapagliflozin on Myocardial index (MI), left ventricular weight (LVW) index and mortality percentage. MI and LVW index values were expressed as mean \pm SD and analyzed using oneway ANOVA followed by the Bonferroni post hoc test for multiple comparisons. Mortality rates were expressed as percentage and analyzed using Pearson chi-square test. Superscript letters indicate a significant difference at $\mathrm{P} \leq 0.05$ (a) Indicates significant differences from control rats. (b) Indicates significant differences from untreated diabetic rats. Myocardial index was calculated using the formula (heart weight to rat weight ratio) * 100, LVW index $=$ (left ventricle weight to heart weight ratio) *100 and Mortality percentage $=$ [number of animals died by the end of the study /starting number of animals per group] *100.

\begin{tabular}{|llll|}
\hline Study groups & $\begin{array}{l}\text { Myocardial } \\
\text { index } \\
(\text { MI) }\end{array}$ & $\begin{array}{l}\text { Left ventricular weight index } \\
(\text { LVW) }\end{array}$ & $\begin{array}{l}\text { Mortality } \\
\text { percentage } \\
(\%)\end{array}$ \\
\hline Control & $0.26 \pm 0.01$ & $22.22 \pm 2.63$ & 0 \\
\hline Untreated diabetic & $0.4 \pm 0.09^{\text {a }}$ & $31.43 \pm 5.86^{\mathrm{a}}$ & $37.5^{\mathrm{a}}$ \\
\hline $\begin{array}{l}\text { Diabetic }+0.75 \mathrm{mg} \\
\text { Dapa }\end{array}$ & $0.27 \pm 0.02^{\mathrm{b}}$ & $18.72 \pm 5.07^{\mathrm{b}}$ & $25^{\mathrm{b}}$ \\
\hline $\begin{array}{l}\text { Diabetic }+1.5 \mathrm{mg} \\
\text { Dapa }\end{array}$ & $0.3 \pm 0.01^{\mathrm{b}}$ & $19.28 \pm 3.41^{\mathrm{b}}$ & $12.5^{\mathrm{b}}$ \\
\hline Diabetic + 3 mg Dapa & $0.28 \pm 0.08^{\mathrm{b}}$ & $22.37 \pm 4.28^{\mathrm{b}}$ & $12.5^{\mathrm{b}}$ \\
\hline
\end{tabular}

\subsection{Dapagliflozin diminished the rise in cardiac enzymes serum levels of diabetic rats}

Serum levels of cardiac enzymes were represented by total lactate dehydrogenase (LDH), Creatine phosphokinase (CPK), Troponin I and creatine kinase-MB isoenzyme (CKMB). All cardiac enzymes were significantly higher in untreated diabetic group comparing with normal control group as shown in Table 3 . Diabetic groups treated with graded doses of Dapa revealed significantly lower serum levels of CPK, CKMB, LDH and Troponin I compared to untreated diabetic group in a dose dependent manner (Table 3). 
Table 3

Effect of graded doses of Dapagliflozin on serum levels of different cardiac enzymes: LDH, CPK, Troponin I and CKMB. All values were expressed as mean \pm SD. Superscript letters indicate a significant difference at $\mathrm{P} \leq 0.05$ using one-way ANOVA followed by the Bonferroni post hoc test for multiple comparisons (a) Indicates significant differences from control rats. (b) Indicates significant differences from untreated diabetic rats. (c) Indicates significant differences from diabetic rats treated with $0.75 \mathrm{mg}$ Dapagliflozin. (d) Indicates significant differences from diabetic rats treated with $1.5 \mathrm{mg}$ Dapagliflozin.

\begin{tabular}{|lllll|}
\hline Study groups & LDH $(\mathrm{pg} / \mathrm{ml})$ & $\mathrm{CPK}(\mathrm{pg} / \mathrm{ml})$ & Troponin I (ng/ml) & CKMB(U/L) \\
\hline Control & $28.6 \pm 2.6^{1}$ & $19.97 \pm 2.47$ & $0.29 \pm 0.03$ & $3.83 \pm 0.18$ \\
\hline Untreated diabetic & $155.2 \pm 8.5^{\mathrm{a}}$ & $121.25 \pm 5.69^{\mathrm{a}}$ & $4 \pm 0.08^{\mathrm{a}}$ & $18.4 \pm 1.34^{\mathrm{a}}$ \\
\hline Diabetic + 0.75 mg Dapa & $99.63 \pm 1.9^{\mathrm{b}}$ & $77.27 \pm 3.48^{\mathrm{b}}$ & $1.54 \pm 0.06^{\mathrm{b}}$ & $9.93 \pm 0.88^{\mathrm{b}}$ \\
\hline Diabetic+ 1.5 mg Dapa & $56.8 \pm 3.6^{\mathrm{b}, \mathrm{c}}$ & $56.87 \pm 2.09^{\mathrm{b}, \mathrm{c}}$ & $0.87 \pm 0.05^{\mathrm{b}, \mathrm{c}}$ & $6.63 \pm 0.29 \mathrm{~b}, \mathrm{c}$ \\
\hline Diabetic+3 mg Dapa & $39.3 \pm 1.2^{\mathrm{b}, \mathrm{c}, \mathrm{d}}$ & $27.43 \pm 0.63^{\mathrm{b}, \mathrm{c}, \mathrm{d}}$ & $0.52 \pm 0.02^{\mathrm{b}, \mathrm{c}, \mathrm{d}}$ & $4.99 \pm 0.07^{\mathrm{b}, \mathrm{c}, \mathrm{d}}$ \\
\hline
\end{tabular}

\subsection{Dapagliflozin elevated the serum levels of erythropoietin}

Serum levels of EPO significantly decreased in untreated diabetic group compared to control group (almost 90\% decreases) as depicted in Fig. 2. However, treatment with graded doses of Dapa could significantly increase serum levels of EPO in a dose dependent manner compared to untreated diabetic group.

\subsection{Dapagliflozin in a dose-dependent manner upregulated the expression of pAkt, pJAK and pMAPK signaling pathways in the heart specimen of diabetic rats}

Figure 3 revealed the effect of graded doses of Dapa $(0.75,1.5$ and $3 \mathrm{mg}, \mathrm{p} .0)$ daily for a month on the expression of pAKT, pJAK and pMAPK in cardiac cells. Statistical analysis of the intensity of the target proteins shows a significant downregulation of pAKT, pJAK and pMAPK in the untreated diabetic group compared to control group. Dapa activated PAKT, pJAK and pMAPK signaling pathways in a dose dependent manner compared to untreated diabetic rats.

\subsection{Dapagliflozin improves histological pictures of the cardiac tissues of diabetic rats}

Sections stained with H\&E of control group showed normal histological structure of the myocardial wall; branched network of cardiac myocytes with central nuclei that separated connective tissue fibres (Fig. 4A). Sections of untreated diabetic group presented focal shrinkage and loss of myofibres with widespread connective tissue elements and leukocytic infiltration. Congested blood vessels as well as 
patches of extravasated blood and interstitial edema were observed (Fig. 4B). Sections of Dapa-treated groups with different doses $(0.75,1.5$ and $3 \mathrm{mg} / \mathrm{kg})$ revealed attenuation of the diabetic-induced histopathological changes; manifested by less edema, congestion and inflammation, in addition to wellorganized cardiomyocytes. (Fig. 4, C, D\&E). Masson trichrome staining sections showed an increase in collagen deposition among the cardiomyocytes in the untreated diabetic group (Fig. 5,B). Dapa-treated groups with different doses significantly attenuated the diabetic-induced increase of collagen deposition (Fig. 5, C, D\&E).

\subsection{Dapagliflozin reduced the immunohistochemical staining of p53 and TNFa in cardiac tissues of Streptozotocin-diabetic rats}

Immunocytochemical activity of cardiac tissue exhibited increase in the immunostaining intensity of p53 (Fig. 6, B) in the diabetic group in comparison to normal control group (Fig. 6, A). Meanwhile, immunoreaction of Dapa-treated groups with different doses $(0.75,1.5 \& 3 \mathrm{mg} / \mathrm{kg})$ displayed weak immunostaining intensity (Fig. 6, C, D \& E) in comparison to the diabetic group. Mean \% area of p53 immunostaining of cardiomyocytes showed marked decrease in the Dapa treated groups with different doses $(0.75,1.5 \& 3 \mathrm{mg} / \mathrm{kg}$ ) in a dose dependent manner (Fig. 6, C, D \&E) compared to the diabetic group. On the other hand, immunostaining of TNF a showed strong reaction of cardiac myocytes of diabetic group (Fig. 7, A\&B). Dapa in a dose dependent manner significantly decreased immunostaining of TNF a (Fig. 7, C, D \& E).

\section{Discussion}

The induction of DCM in rats by high fat diet and STZ resulted in various alterations that mimic the clinical picture of DCM patients. The present study depicted an electrocardiographic weakness of Voltage of $\mathrm{R}$ in the untreated diabetic group. Voltage of $\mathrm{R}$ is a measure of power of contractility of the heart. It was reported that increase of collagen regions in the cardiomyocytes during DCM leads to disturbance of excitation-contraction coupling between cardiomyocytes, increased stiffness of the myocardium and decreased in the heart contractility [8]. In addition, compensatory cardiac hypertrophy mechanism during DCM to keep pace with increased functional demand, normalize increased stress in the ventricular wall and affect the contractile capacity of the heart [9]. Previous work demonstrated that myocardial inflammation may directly affect cardiac contractility [10]. TNF-a exerts a negative inotropic effect on the heart, inducing a rapid and reversible contractile dysfunction [10]. Prolonged QT interval in diabetic rats is an indicated marker of increased risk of ventricular arrhythmias and heart failure. Additionally, the exaggerated release of positively charged $\mathrm{Ca}^{++}$ions in cardiomyocytes during DCM may cause irregular repolarization, which could explain the QT interval prolongation in diabetic rats. Abnormally deep Q wave found in our results could reflect the hypertrophic cardiomyopathy with expected loss of local electrical forces due to myocardial fibrosis or ventricular hypertrophy [11]. Daily administration of Dapa for 4 weeks attenuated all ECG parameters abnormalities in untreated diabetic rats in a dose dependent manner in terms of decreased QT interval prolongation, rendered voltage of R to normal, decreased ST segment 
elevation and decreased percentage of pathological Q waves. This amelioration by Dapa can be attributed to suppression of prolonged ventricular-repolarization through improvement of mitochondrial function and alleviating of oxidative stress [12]. Moreover, it was reported that activation of Akt signalling increases cell size and contractile efficiency [9] and this was confirmed by our present results. The cardioprotective effect of Dapa in this study as noted by decreasing ST segment elevation may attribute to its ability to decrease apoptosis in the cardiomyocytes [13]. This is confirmed in the current study by the ability of Dapa to cause a marked decrease in p53 protein in the cardiomyocytes thus decreasing apoptosis. In addition, Dapa in the current study rendered the $\mathrm{Q}$ wave to normal, this may be explained by its ability to reduce left ventricular hypertrophy and decrease LV mass weight by increasing glucose excretion, decreasing body weight, inflammation, preload and afterload, infract size, hyperinsulinemia and insulin resistance [13].

Diabetes induces both collagen deposition and cross-linking resulting in compromised ventricular compliance [14]. In addition, hyperinsulinemia and insulin resistance can cause cardiomyocyte hypertrophy by different mechanisms; one of them is the increase in Brain natriuretic peptide (BNP). BNP is a biomolecule released from the ventricles in response to myocardial stretch and is an important marker for cardiomyocytes hypertrophy. Furthermore, increase of inflammatory cytokines in the diabetic heart was found to promote cardiac derangement by modulating certain intracellular signalling pathways in cardiac cells. This can lead to cardiomyocyte hypertrophy, death, and cardiac fibrosis [15]. It was illustrated that continuous activation of TNF signalling motivates cardiomyocyte apoptosis and remodelling by activation of both intrinsic and extrinsic cell death pathways, with resultant increases of cytosolic levels of cardiac enzymes leading to increase their serum levels .

Untreated diabetic rats showed focal shrinkage, loss of myofibers, leukocytic infiltration, congested blood vessels, patches of extravasated blood (hemorrhage) and interstitial edema. Inflammation can lead to fibrosis, cell death and cardiac remodelling. Fibrosis and hypertrophy mediate diastolic stiffness which is a hallmark of the diabetic heart. Here in this study, Dapa attenuated diabetic-induced histopathological changes; manifested by less edema and congestion.

Masson trichrome staining of the cardiomyocytes of untreated diabetic rats in our study revealed an increase in the collagen deposition. Myocardial fibrosis is characterized by accumulation of activated fibroblasts and excessive deposition of fibrotic extracellular matrix proteins, especially type I collagen. One explanation for fibrosis in DCM was increasing cytokines and pro-fibrotic factors released by cardiac cells and inflammatory cells. TNF-a is one of the inflammatory cytokines that can directly stimulates cardiac fibroblast proliferation and collagen production. Therefore, its rise results in cardiomyocyte apoptosis and remodelling [15]. Moreover, this explained the increase of immunostaining intensity of TNF in the STZ induced diabetic group in our study. One more explanation for this result is that hyperglycaemia has a direct relationship with the progress of inflammation revealed by the increased expression of proinflammatory cytokines such as IL-6, TNF- $a$, and NF $\kappa$ B. However, Dapa attenuated the diabetic-induced increase of collagen deposition after 4 weeks treatment. Dapa also displayed a significant reduction in immunostaining intensity of TNF compared to the diabetic group. In the current

Page $11 / 23$ 
study, STZ induced diabetic rats showed increase immunostaining intensity of p53 in the heart tissues and this is in line with previous studies $[16,17]$. This may be explained by a study indicated that increased ROS production in oxidative stress results in cardiomyocytes apoptosis in rats during DCM [18]. On the other hand, Dapa attenuated the apoptosis by decreasing p53 levels in cardiac cells. This is in accordance with previous study showed that Dapa protected cardiomyocytes against apoptosis by decreasing infract size and increasing $\mathrm{Bcl} 2$ which in return increases anti apoptotic protein expression and attenuated mitochondrial dysfunction in the heart by decreasing ROS production [19]. Furthermore, Dapa had a direct antioxidant effect on the heart decreasing the oxidative stress consequently decreasing apoptosis in cardiac cells [20].

The current study showed highly significant decrease in EPO levels in type 2 diabetic rats. The deficiency in EPO levels in diabetes has been proposed for various mechanisms; including abnormal anaemia sensing mechanisms due to diabetic autonomic neuropathy, impaired production of EPO owing to tubulointerstitial damage, and dysfunction of hypoxia inducible factor (HIF). A study suggested that the elevation of inflammatory cytokines could trigger diabetic kidney disease and anaemia and have anti erythropoietic effect that may change the sensitivity of progenitors to erythropoietin [21]. It also promotes the apoptosis of immature erythrocytes resulting in a further decrease in the number of circulating erythrocytes [21]. Eventually, EPO deficiency in diabetes was attributed to development of nephropathy and kidney diseases [22]. In our study, Dapa in a dose dependent manner showed a significant rise in EPO serum levels. That may be contributed to its nephroprotective effect and lowering the progression of chronic kidney diseases [23].

Another possible illustration for the increased EPO levels is that Dapa may exacerbate hypoxia in the renal medulla by activating sodium reuptake at the thick ascending limb, leading to improved oxygen supply-demand balance in the renal cortex. This may stimulate more EPO production [24] .

Binding of EPO with its receptor, simultaneously activates three major transduction pathways including, the Janus-activated kinase- signal transducer and activator of transcription (JAK2-STAT5), phosphatidylinositol-3-kinase-Akt (PI3K-Akt), and extracellular signal-related kinase (ERK) MAPK cascades, that which results in proliferation and differentiation of cells and decreases apoptosis and heart failure in diabetic patients. In the current study, marked suppression of pAKT, pJAK and pMAPK signalling pathways in the cardiac cells were observed in STZ induced diabetic rats. Thus, this can be partly explained by the reduction of EPO levels. In type 2 diabetes, insulin-mediated activation of PI3K/AKT pathway is blocked [25]. Dysregulation of Jak pathway was reported in patients with cardiomyopathy [26] and this may be explained by the rise of inflammatory cytokines [27]. Here, Dapa reduced significantly the expression of TNF-a and increased the levels of EPO and hence upregulated the expression of pAKT, pJAK and pMAPK signalling pathways in the cardiac cells of diabetic rats.

To conclude, Dapa had a promising cardioprotective effect by increasing EPO serum levels, consequently activation of expression and signalling pathways of pAKT, pJAK2 and pMAPK cascades. This results in 
proliferation and differentiation of heart cells and decreases apoptosis. Using Dapa in diabetic patients promises to substantially reduce cardiovascular complications and improve patient quality of life.

\section{Declarations}

\section{Ethics approval and consent to participate}

Not applicable

\section{Consent to participate}

Not applicable

\section{Availability of data and materials}

The data that support the findings of this study are available on request from the corresponding author.

\section{Authors' contributions}

Nora El-Sayed: Investigation, Methodology, Writing - Original Draft, Visualization

Yasser M. A. Moustafa : Supervision, Writing - Review \& Editing

Noha AboGresha : Investigation, Methodology, Writing - Review \& Editing

Amal A.M. Ahmed: Investigation, Methodology Writing - Review \& Editing

Islam Mahmoud : Investigation, Methodology, Writing - Review \& Editing

Norhan M El-Sayed: Conceptualization, Writing - Review \& Editing, Formal analysis

\section{Declaration of interests}

$\bigotimes \sqrt{ }$ The authors declare that they have no known competing financial interests or personal relationships that could have appeared to influence the work reported in this paper.

\section{Funding sources}

This research did not receive any specific grant from funding agencies in the public, commercial, or notfor-profit sectors.

\section{Compliance with Ethical Standards}

\section{Disclosure of potential conflicts of interest}

The authors declared that there are no conflicts of interest. 


\section{Research involving Animals}

This study was carried out according to the "Guidelines of the Animal Care and Use of laboratory animals

" and in parallel with the guidelines adopted by Research Committee at Suez Canal University (approval no. 201812MA2).

\section{Informed consent}

Not applicable

\section{References}

1. Jia G, Hill MA, Sowers JR. Diabetic Cardiomyopathy: An Update of Mechanisms Contributing to This Clinical Entity. Circulation research. 2018;122(4):624-38. doi:10.1161/CIRCRESAHA.117.311586.

2. Fujita Y, Doi Y, Hamano T, Hatazaki M, Umayahara Y, Isaka Y, Tsubakihara Y. Low erythropoietin levels predict faster renal function decline in diabetic patients with anemia: a prospective cohort study. Scientific reports. 2019;9(1):14871. doi:10.1038/s41598-019-51207-8.

3. Symeonidis A, Kouraklis-Symeonidis A, Psiroyiannis A, Leotsinidis M, Kyriazopoulou V, Vassilakos P, Vagenakis A, Zoumbos N. Inappropriately low erythropoietin response for the degree of anemia in patients with noninsulin-dependent diabetes mellitus. Annals of hematology. 2006;85(2):79-85. doi:10.1007/s00277-005-1102-9.

4. Shi Z, Hodges VM, Dunlop EA, Percy MJ, Maxwell AP, El-Tanani M, Lappin TR. Erythropoietin-induced activation of the JAK2/STAT5, PI3K/Akt, and Ras/ERK pathways promotes malignant cell behavior in a modified breast cancer cell line. Molecular cancer research: MCR. 2010;8(4):615-26. doi:10.1158/1541-7786.MCR-09-0264.

5. Wiviott SD, Raz I, Bonaca MP, Mosenzon O, Kato ET, Cahn A, Silverman MG, Zelniker TA, Kuder JF, Murphy SA, Bhatt DL, Leiter LA, McGuire DK, Wilding JPH, Ruff CT, Gause-Nilsson IAM, Fredriksson M, Johansson PA, Langkilde AM, Sabatine MS, Investigators D-T. Dapagliflozin and Cardiovascular Outcomes in Type 2 Diabetes. N Engl J Med. 2019;380(4):347-57. doi:10.1056/NEJMoa1812389.

6. Vatandoust N, Rami F, Salehi AR, Khosravi S, Dashti G, Eslami G, Momenzadeh S, Salehi R. Novel High-Fat Diet Formulation and Streptozotocin Treatment for Induction of Prediabetes and Type 2 Diabetes in Rats. Advanced biomedical research. 2018;7:107. doi:10.4103/abr.abr_8_17.

7. Darwesh AM, El-Azab MF, Abo-Gresha NM, El-Sayed NM, Moustafa YM. Cardioprotective Mechanisms of Exenatide in Isoprenaline-induced Myocardial Infarction: Novel Effects on Myocardial alpha-Estrogen Receptor Expression and IGF-1/IGF-2 System. J Cardiovasc Pharmacol. 2018;71(3):160-73. doi:10.1097/FJC.0000000000000557.

8. Menon SC, Eidem BW, Dearani JA, Ommen SR, Ackerman MJ, Miller D. Diastolic dysfunction and its histopathological correlation in obstructive hypertrophic cardiomyopathy in children and adolescents. Journal of the American Society of Echocardiography: official publication of the American Society of Echocardiography. 2009;22(12):1327-34. doi:10.1016/j.echo.2009.08.014. 
9. Harvey PA, Leinwand LA. The cell biology of disease: cellular mechanisms of cardiomyopathy. J Cell Biol. 2011;194(3):355-65. doi:10.1083/jcb.201101100.

10. Van Tassell BW, Seropian IM, Toldo S, Mezzaroma E, Abbate A. Interleukin-1beta induces a reversible cardiomyopathy in the mouse. Inflammation research: official journal of the European Histamine Research Society [et al]. 2013;62(7):637-40. doi:10.1007/s00011-013-0625-0.

11. Koga $Y$, Yamaga A, Hiyamuta $K$, Ikeda $H$, Toshima $H$. Mechanisms of abnormal $Q$ waves in hypertrophic cardiomyopathy assessed by intracoronary electrocardiography. J Cardiovasc Electrophys. 2004;15(12):1402-8. doi:10.1046/j.1540-8167.2004.04314.x.

12. Durak A, Olgar Y, Degirmenci S, Akkus E, Tuncay E, Turan B. A SGLT2 inhibitor dapagliflozin suppresses prolonged ventricular-repolarization through augmentation of mitochondrial function in insulin-resistant metabolic syndrome rats. Cardiovascular diabetology. 2018;17(1):144. doi:10.1186/s12933-018-0790-0.

13. Lahnwong S, Chattipakorn SC, Chattipakorn N. Potential mechanisms responsible for cardioprotective effects of sodium-glucose co-transporter 2 inhibitors. Cardiovascular diabetology. 2018;17(1):101. doi:10.1186/s12933-018-0745-5.

14. Li CJ, Lv L, Li H, Yu DM. Cardiac fibrosis and dysfunction in experimental diabetic cardiomyopathy are ameliorated by alpha-lipoic acid. Cardiovascular diabetology. 2012;11:73. doi:10.1186/14752840-11-73.

15. Frati G, Schirone L, Chimenti I, Yee D, Biondi-Zoccai G, Volpe M, Sciarretta S. An overview of the inflammatory signalling mechanisms in the myocardium underlying the development of diabetic cardiomyopathy. Cardiovascular research. 2017;113(4):378-88. doi:10.1093/cvr/cvx011.

16. Al-Ani B, Alzamil NM, Hewett PW, Al-Hashem F, Bin-Jaliah I, Shatoor AS, Kamar SS, Latif NSA, Haidara MA, Dawood AF. (2021) Metformin ameliorates ROS-p53-collagen axis of fibrosis and dyslipidemia in type 2 diabetes mellitus-induced left ventricular injury. Arch Physiol Biochem:1-7. doi:10.1080/13813455.2020.1869265.

17. Gu J, Wang S, Guo H, Tan Y, Liang Y, Feng A, Liu Q, Damodaran C, Zhang Z, Keller BB, Zhang C, Cai L. Inhibition of p53 prevents diabetic cardiomyopathy by preventing early-stage apoptosis and cell senescence, reduced glycolysis, and impaired angiogenesis. Cell death disease. 2018;9(2):82. doi:10.1038/s41419-017-0093-5.

18. Liu ZW, Zhu HT, Chen KL, Dong X, Wei J, Qiu C, Xue JH. Protein kinase RNA-like endoplasmic reticulum kinase (PERK) signaling pathway plays a major role in reactive oxygen species (ROS)mediated endoplasmic reticulum stress-induced apoptosis in diabetic cardiomyopathy. Cardiovascular diabetology. 2013;12:158. doi:10.1186/1475-2840-12-158.

19. Lahnwong S, Palee S, Apaijai N, Sriwichaiin S, Kerdphoo S, Jaiwongkam T, Chattipakorn SC, Chattipakorn N. Acute dapagliflozin administration exerts cardioprotective effects in rats with cardiac ischemia/reperfusion injury. Cardiovascular diabetology. 2020;19(1):91. doi:10.1186/s12933-020-01066-9. 
20. Arow M, Waldman M, Yadin D, Nudelman V, Shainberg A, Abraham NG, Freimark D, Kornowski R, Aravot D, Hochhauser E, Arad M. Sodium-glucose cotransporter 2 inhibitor Dapagliflozin attenuates diabetic cardiomyopathy. Cardiovascular diabetology. 2020;19(1):7. doi:10.1186/s12933-019-09804.

21. Barbieri J, Fontela PC, Winkelmann ER, Zimmermann CE, Sandri YP, Mallet EK, Frizzo MN. (2015) Anemia in Patients with Type 2 Diabetes Mellitus. Anemia 2015:354737. doi:10.1155/2015/354737.

22. Jha V, Garcia-Garcia G, Iseki K, Li Z, Naicker S, Plattner B, Saran R, Wang AY, Yang CW. Chronic kidney disease: global dimension and perspectives. Lancet. 2013;382(9888):260-72. doi:10.1016/S01406736(13)60687-X.

23. Liuzzo G, Patrono C. Management of chronic kidney disease and its cardiovascular complications: has the dawn of a new era arrived? Comment on 'Dapagliflozin in Patients with Chronic Kidney Disease'. European heart journal. 2020;41(44):4231-2. doi:10.1093/eurheartj/ehaa890.

24. O'Neill J, Fasching A, Pihl L, Patinha D, Franzen S, Palm F. Acute SGLT inhibition normalizes 02 tension in the renal cortex but causes hypoxia in the renal medulla in anaesthetized control and diabetic rats. American journal of physiology Renal physiology. 2015;309(3):F227-34. doi:10.1152/ajprenal.00689.2014.

25. Huang X, Liu G, Guo J, Su Z. The PI3K/AKT pathway in obesity and type 2 diabetes. Int J Biol Sci. 2018;14(11):1483-96. doi:10.7150/ijbs.27173.

26. Podewski EK, Hilfiker-Kleiner D, Hilfiker A, Morawietz H, Lichtenberg A, Wollert KC, Drexler H.

Alterations in Janus kinase (JAK)-signal transducers and activators of transcription (STAT) signaling in patients with end-stage dilated cardiomyopathy. Circulation. 2003;107(6):798-802.

doi:10.1161/01.cir.0000057545.82749.ff.

27. Kishore R, Verma SK. Roles of STATs signaling in cardiovascular diseases. Jak-Stat. 2012;1(2):11824. doi:10.4161/jkst.20115.

\section{Figures}


(A)
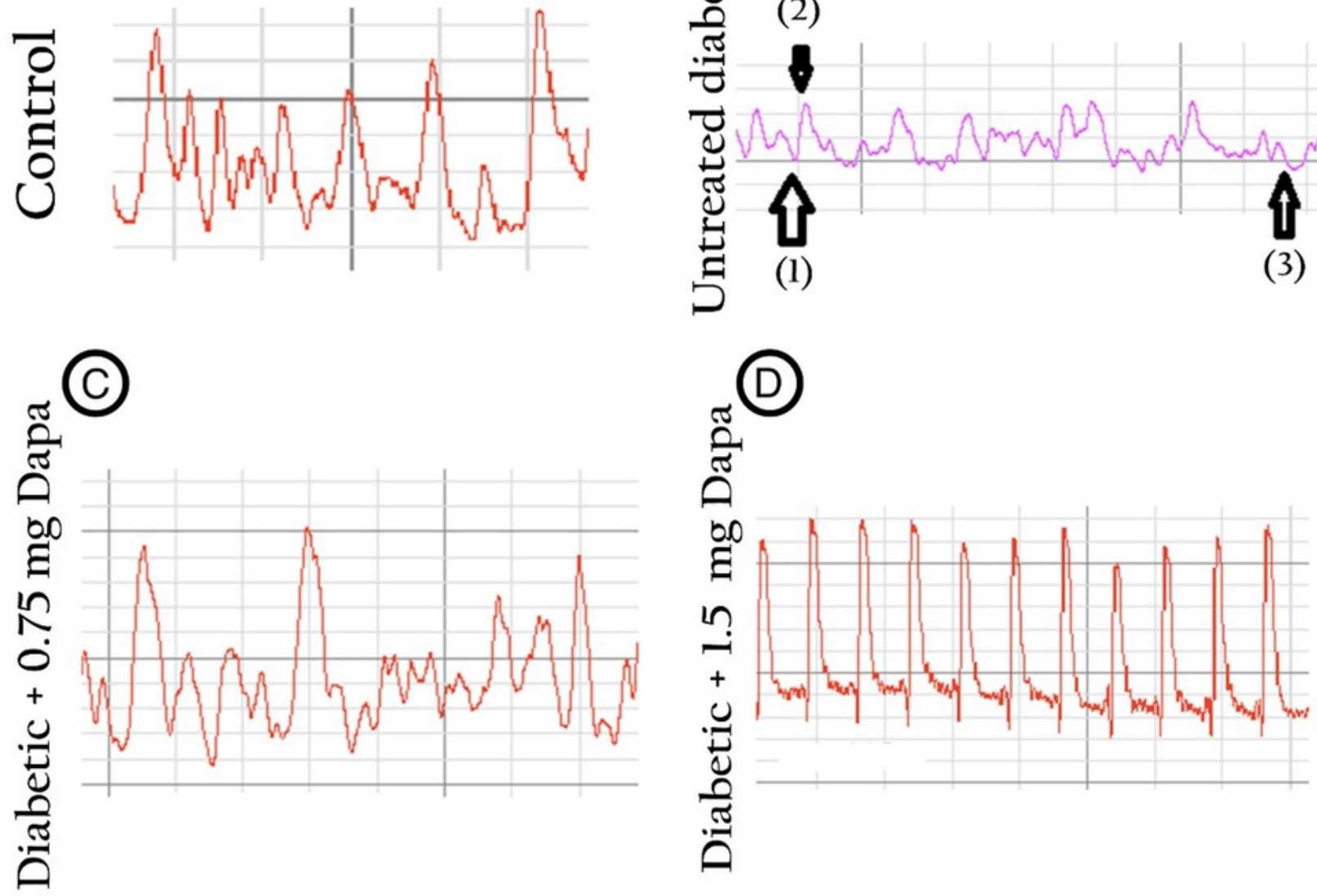

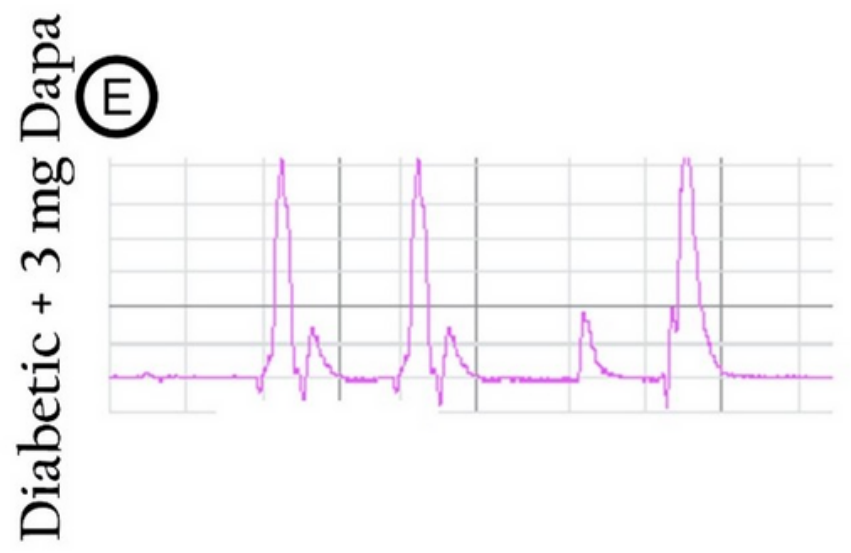

Figure 1

Effect of graded doses of Dapagliflozin on ECG recordings in diabetic rats. (A) control group. (B) Untreated diabetic group showing abnormalities in all ECG parameters including deep pathological Q wave (arrow 1), weak contractility of voltage of R (arrow 2), wide pathological Q wave (arrow 3), QT interval prolongation and ST segment elevation. Dapagliflozin treated groups with three different graded doses, (C) Dapa 0.75mg/kg, (D) Dapa 1.5mg/kg and (E) Dapa $3 \mathrm{mg} / \mathrm{kg}$ 


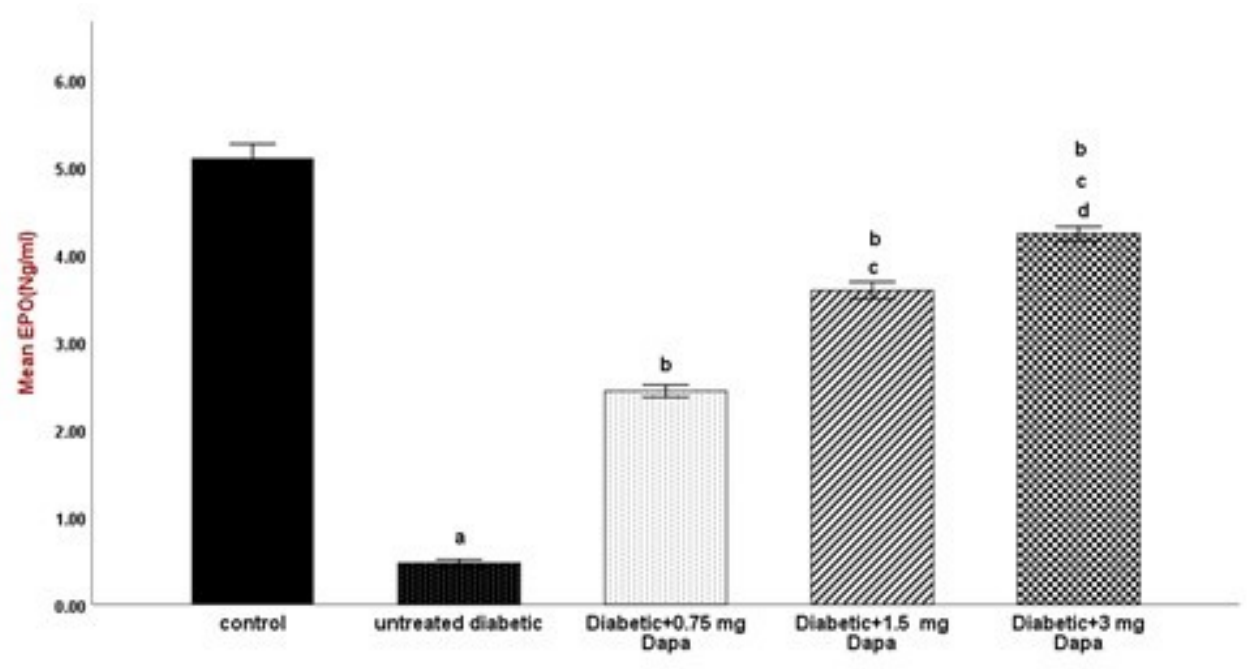

Figure 2

Effect of different doses of Dapagliflozin on Serum levels of EPO. (a) Indicates significant differences from control rats. (b) Indicates significant differences from untreated diabetic rats. (c) Indicates significant differences from diabetic rats treated with $0.75 \mathrm{mg}$ Dapa.(d) Indicates significant differences from diabetic treated rats with $1.5 \mathrm{mg}$ Dapa. All values were expressed as mean $\pm S D$. Superscript letters indicate a significant difference at $\mathrm{P} \leq 0.05$ using one-way ANOVA followed by the Bonferroni post hoc test for multiple comparisons.

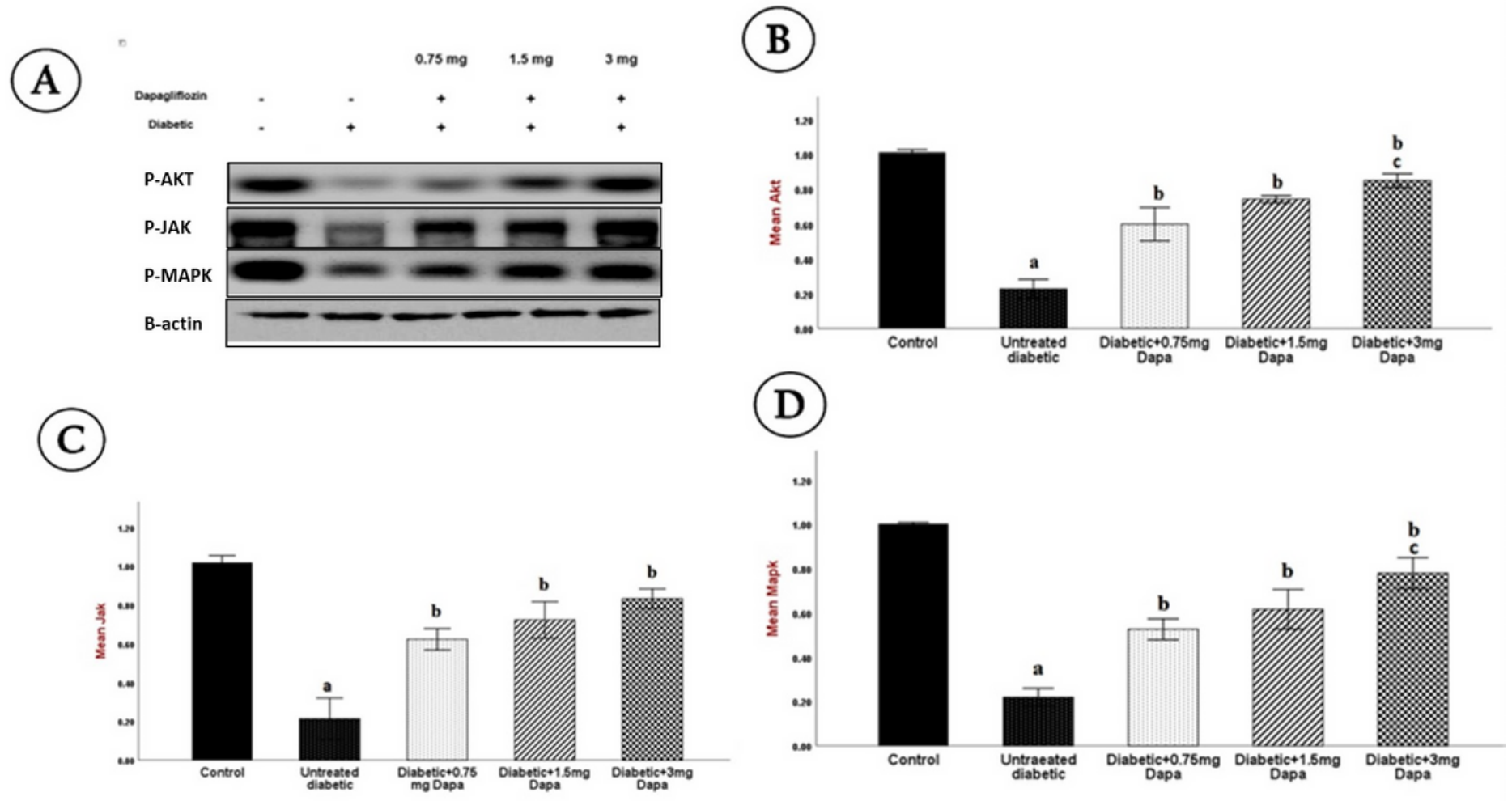

Figure 3 
Effect of different doses of Dapagliflozin on the expression of pAKT, pJAK and pMAPK. (A) Heart cells from different experimental groups were lysed and the proteins separated by SDS-PAGE and western blotted using antisera against against pAKT, pJAK and pMAPK. $\beta$-actin was used as a loading control. (B) Quantified data of pAKT normalized to $\beta$-actin. (C) Quantitated data of pJAK normalized to $\beta$-actin. (D) Quantitated data of pMAPK normalized to $\beta$-actin. All values are expreesed as mean+SD. Superscript letters indicate a significant difference at $\mathrm{P} \leq 0.05$ using one-way ANOVA followed by the Bonferroni post hoc test for multiple comparisons. (a) Indicates significant differences from control rats. (b) Indicates significant differences from untreated diabetic rats. (c) Indicates significant differences from diabetic group treated with $0.75 \mathrm{mg}$ Dapagliflozin.
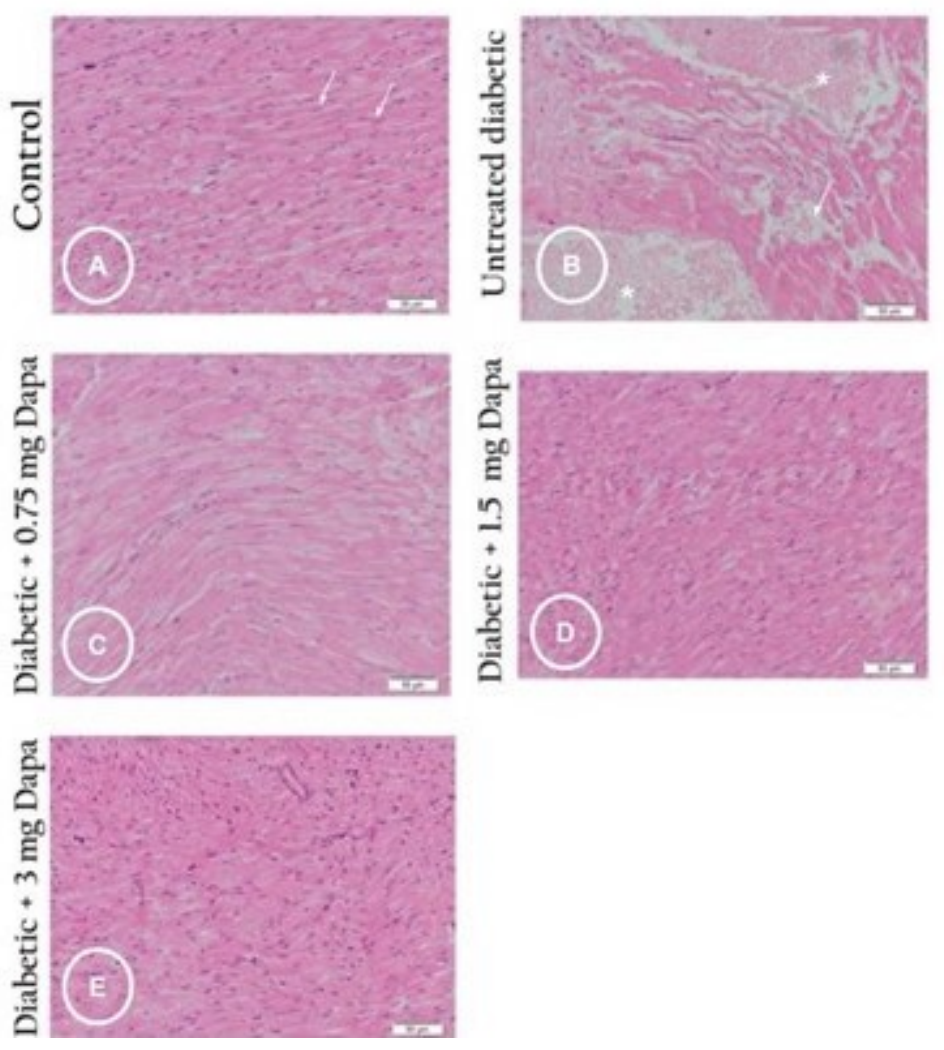

\section{Figure 4}

Photomicrographs of examined heart tissues of different groups stained with H\&E.(A) control group showing normal structure of cardiac wall with branched myofibres and central nuclei (white arrows). (B) Untreated diabetic group showing shrinkage of the cardiac myofibres, congested blood vessels $(*)$, leukocytic infiltration and edema (white arrow). Dapagliflozin treated groups with three different graded doses (C) at dose of $0.75 \mathrm{mg} / \mathrm{kg}$, (D) at dose of $1.5 \mathrm{mg} / \mathrm{kg}$ and (E) at dose of $3 \mathrm{mg} / \mathrm{kg}$ showing attenuation of Histological changes, organized cardiac myofibres and less congestion and inflammatory cell infiltration. Scale bar $50 \mu \mathrm{m}$. 

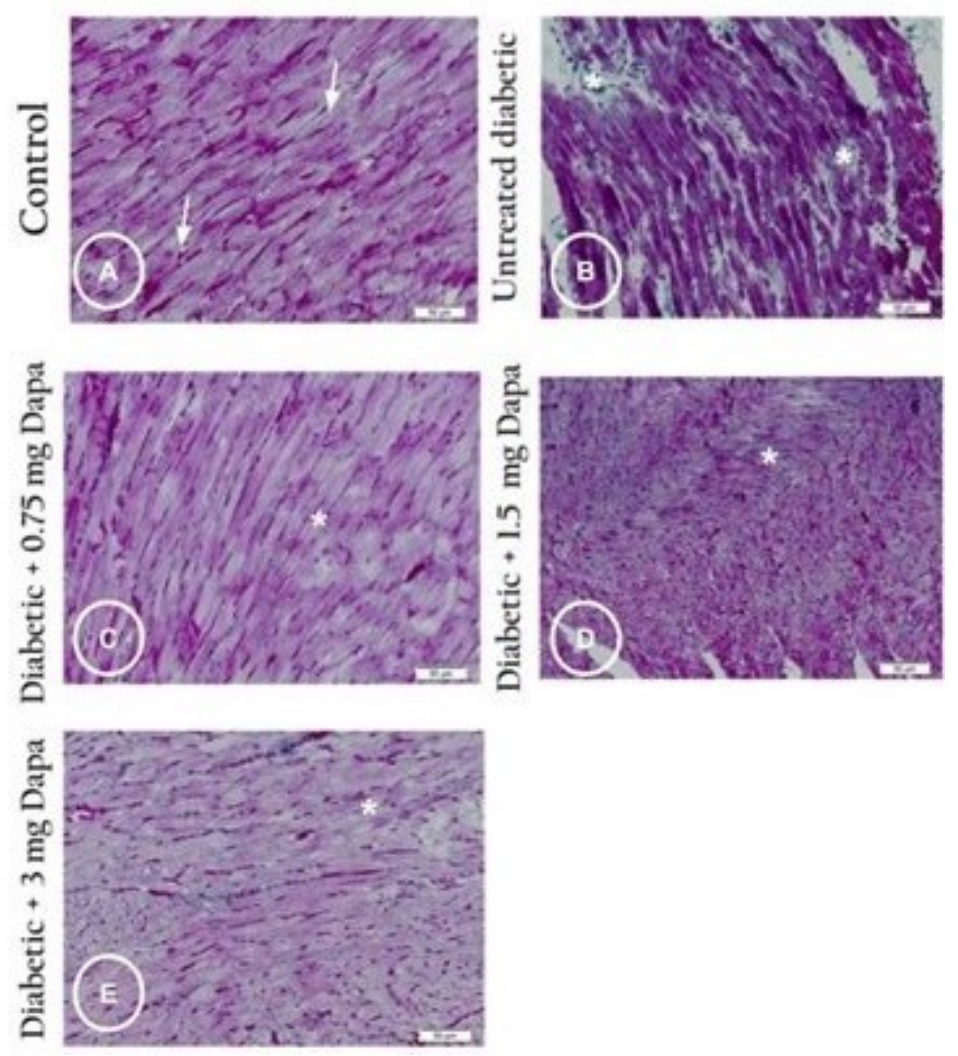

Figure 5

Photomicrographs of cardiac tissues of different groups stained with Masson's trichrome. (A) Control group showing normal histological structure of the heart tissue, branched and anastomosed cardiac fibres (white arrows). (B) Untreated diabetic group showing intense collagen fibres deposition (*). Dapagliflozin treated rats with three different graded doses, (C) at dose of $0.75 \mathrm{mg} / \mathrm{kg}$, (D) at dose of $1.5 \mathrm{mg} / \mathrm{kg}$ and $(\mathrm{E})$ at dose of $3 \mathrm{mg} / \mathrm{kg}$ showing attenuation of collagen fibre distribution $\left.{ }^{*}\right)$. Scale bar $50 \mu \mathrm{m}$. 

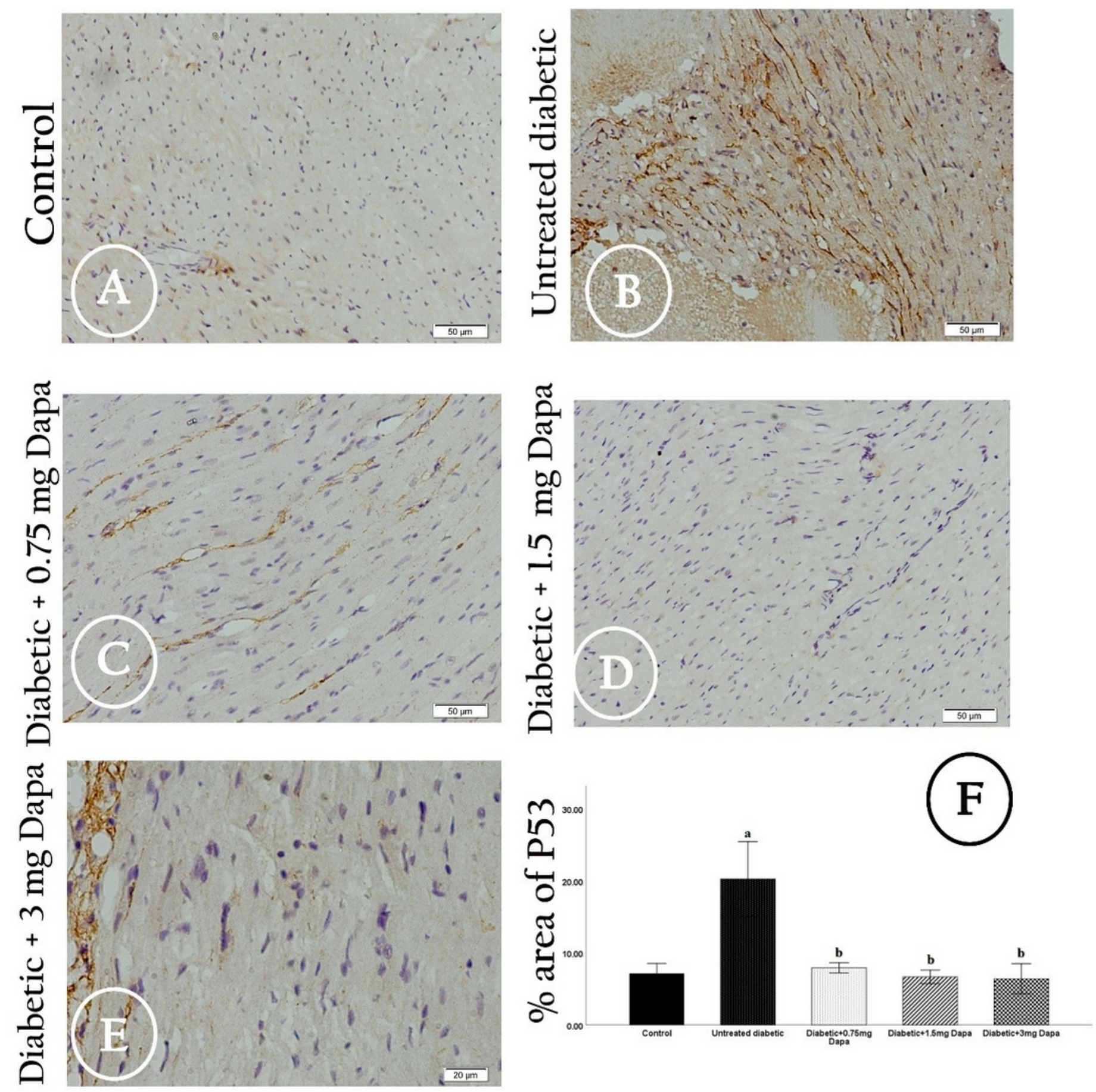

\section{Figure 6}

Photomicrographs showing immunostaining of p53 in the myocardium of experimental groups. (A) Control group. (B) Untreated diabetic group. Dapagliflozin treated groups with three different graded doses, (C) at dose of $0.75 \mathrm{mg} / \mathrm{kg}$, (D) at dose of $1.5 \mathrm{mg} / \mathrm{kg}$ and $(E)$ at dose of $3 \mathrm{mg} / \mathrm{kg}$. (F) Mean percentage area of immunostaining reactivity of p53 in myocardium cells. All values were expressed as mean \pm SD. Superscript letters indicate a significant difference at $P \leq 0.05$ using one-way ANOVA followed 
by the Bonferroni post hoc test for multiple comparisons. (a) Indicates significant differences from

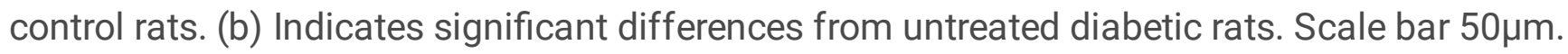
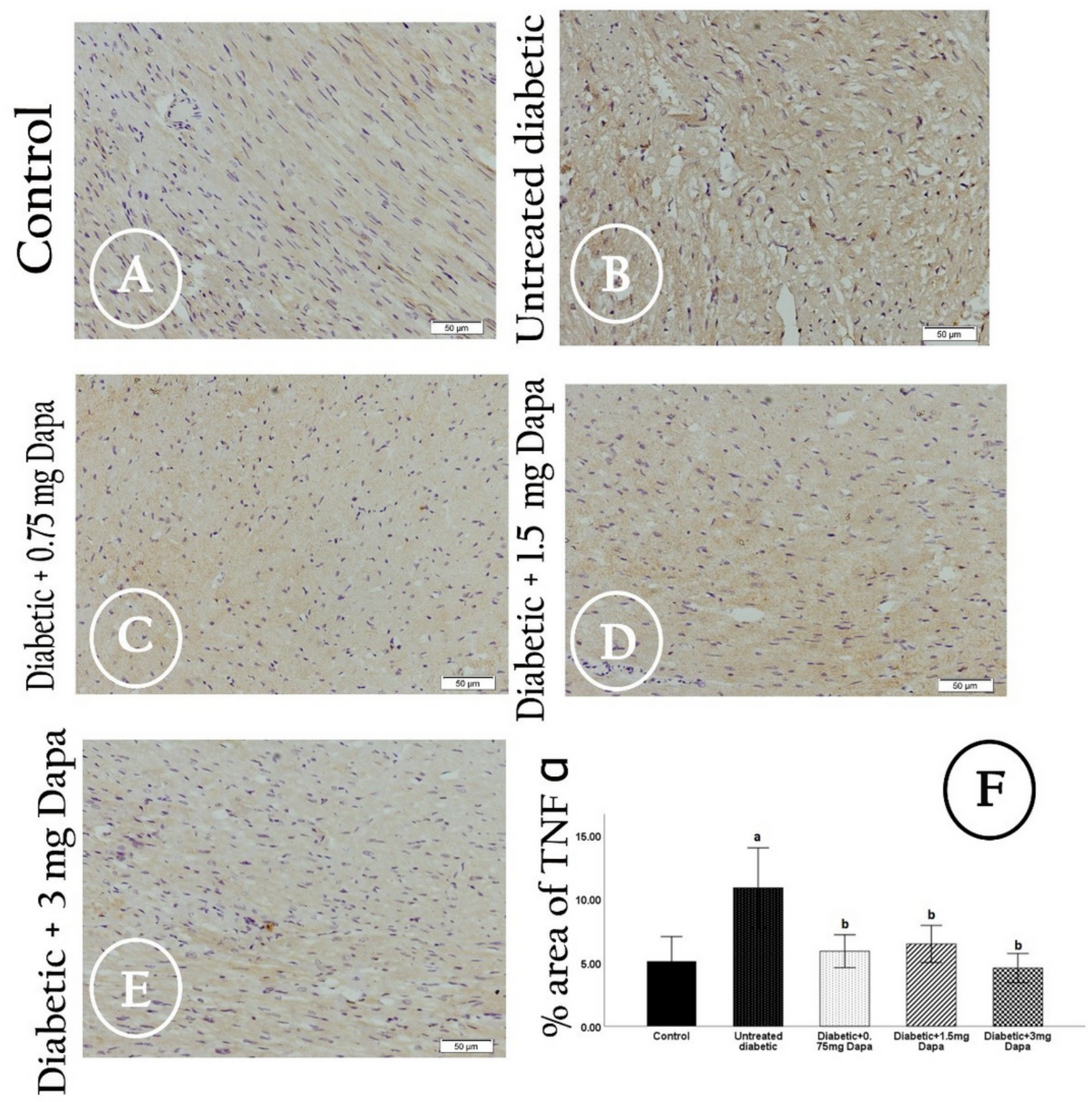

\section{Figure 7}

Photomicrographs showing immunostaining of TNF-a in the myocardium of experimental groups. (A) Control group. (B) Untreated diabetic group. Dapagliflozin treated groups with three different graded doses, (C) at dose of $0.75 \mathrm{mg} / \mathrm{kg}$, (D) at dose of $1.5 \mathrm{mg} / \mathrm{kg}$ and $(E)$ at dose of $3 \mathrm{mg} / \mathrm{kg}$. (F) Mean percentage area of immunostaining reactivity of TNF-a in myocardium cells. All values were expressed as mean \pm SD. Superscript letters indicate a significant difference at $P \leq 0.05$ using one-way ANOVA followed 
by the Bonferroni post hoc test for multiple comparisons. (a) Indicates significant differences from control rats. (b) Indicates significant differences from untreated diabetic rats. Scale bar $50 \mu \mathrm{m}$. 